\title{
Designing a functional rice muffin formulated with prebiotic oligosaccharides and sugar reduction
}

\author{
Cláudia Amorim ${ }^{a, *}$, Beatriz B. Cardoso ${ }^{\text {a }}$, Sara C. Silvério ${ }^{\text {a }}$, Jessica C. Silva ${ }^{\text {a }}$, Joana I. Alves ${ }^{\text {a }}$, \\ Maria Alcina Pereira ${ }^{a}$, Ramón Moreira ${ }^{b}$, Lígia R. Rodrigues ${ }^{a}$ \\ ${ }^{a}$ CEB-Centre of Biological Engineering, Universidade do Minho, Campus de Gualtar, 4710-057, Braga, Portugal \\ ${ }^{\mathrm{b}}$ Chemical Engineering Department, Universidade de Santiago de Compostela, Campus Vida, 15782, Santiago de Compostela, Spain
}

\section{A R T I C L E I N F O}

\section{Keywords:}

Prebiotic

Inulin

Galacto-oligosaccharides

Novel food

Rice muffin

\begin{abstract}
A B S T R A C T
Innovation of pastry products towards higher nutritional and commercial value remains a challenge to the growing field of healthy food. In this study, the prebiotic supplementation and sugar reduction were explored in a widely consumed pastry product with a low level of innovation. The prebiotic potential of commercial agave inulin and galacto-oligosaccharides (GOS) was evaluated and compared by an in vitro model using human fecal inocula. Rice muffins containing $100 \%$ of sugar or $75 \%$ of sugar supplemented with $0.8 \%$ GOS were produced and compared with commercial rice muffins regarding their physical and textural properties. GOS fermentation led to the highest production of lactate and short-chain fatty acids, besides the most significant reduction of the final $\mathrm{pH}$ value and of the ammonia and methane production. Inulin presented a higher selectivity towards Lactobacillaceae ( $51 \pm 1 \%$ of all), while GOS are more efficient to stimulate Bifidobacteriaceae growth ( $65 \pm 7 \%$ of all).

Both prebiotics were effective in reducing Methanobacteria. The reduction of sugar content modified the air bubbles characteristics (size and population with a greater number of tunnels) present in the muffin crumb, without relevant differences in apparent porosity. Textural results indicated that springiness and resilience of the muffin with low sugar content are acceptable, but hardness and chewiness were increased. The new-formula muffins presented very relevant textural parameters with comparable values to those reported in the literature for the commercial ones, thus anticipating a good consumer acceptance. This study is an important contribution towards more innovative and diversified healthy pastry products.
\end{abstract}

\section{Introduction}

The increasing prevalence of overweight and obesity is associated with many diet-related chronic diseases. In this sense, consumers today are pivoting their preferences towards healthier options, such as lowsugar, low-fat and functional food products. The latter are characterized for containing functional ingredients (e.g. prebiotics) that will confer extra health benefits above the original nutritional value of the food product (Mohanty et al., 2018; Szutowska, 2020). In this context, prebiotics have attracted global attention due to their beneficial effects over different physiological functions. These compounds have been reported as promoters of beneficial effects on both human and animal health, namely on the gastrointestinal (GI) tract, cardiometabolism, mental health and bones, among others (Devaraj et al., 2020; Gibson et al., 2017; Sreenivas \& Lele, 2013). According to the updated definition by The International Scientific Association for Probiotics and Prebiotics (ISAPP), a prebiotic is "a substrate that is selectively utilized by host microorganisms conferring a health benefit" (Gibson et al., 2017). In addition, these compounds can be fermented by intestinal bacteria leading to the production of short-chain fatty acids (SCFA) and gases that beneficially modulate the gut environment (Amorim et al., 2020a, 2020b).

The global market of the prebiotic ingredients is expanding with a forecasted compound annual growth rate (CAGR) of 9.8\% (2019-2024) (Ahuja \& Mamtani, 2019). On the other hand, pastry products are widely consumed by people throughout the world. For instance, the European market of bakery products, including muffins and pastries, is expected to grow at a CAGR of $3.12 \%(2020-2025)$ (Mordor Intelligence, 2020). However, there is a lack of innovation in the traditional pastry sector, namely regarding the delivery of healthier alternative

\footnotetext{
* Corresponding author.

E-mail address: claudia.oliveira.amorim@ceb.uminho.pt (C. Amorim).
} 
products.

Prebiotic oligosaccharides can be used as low-calorie sweeteners, presenting favorable organoleptic properties, temperature and acidic stability, which make them potential food ingredients. Inulin and galacto-oligosaccharides (GOS) are commercially well-established prebiotics, recognized as safe food ingredients by the European Food Safety Authority and have been used in the European Union over two decades (Amorim et al., 2019). Inulin is comprised by water-soluble oligo- and polysaccharides consisting of fructose moieties linked to each other through $\beta-(2 \rightarrow 1)$ linkages and to a terminal glucose residue by $\alpha$-linkages (Ahmed \& Rashid, 2019). GOS are composed of galactose (Gal) moieties linked to each other and terminally connected to a glucose (Glc) residue via $\beta$-linkages according to the formula Galn-Glc, with a degree of polymerization (DP) varying from 2 to 8 (Torres et al., 2010).

In this study, an in vitro model using human fecal inocula and highthroughput sequencing (16S rRNA gene) of the microbiota was used to evaluate and compare the prebiotic potential of commercial agave inulin and GOS. Unlike the in vivo studies, the in vitro approach using fecal inocula is a cheap and fast methodology to evaluate the prebiotic potential in a preliminary way (Date et al., 2014). The use of fecal inocula and high-throughput sequencing techniques to analyse the microbiota modulation is compatible and well-aligned with the guidelines stated for the in vitro studies by ISAPP (Gibson et al., 2017). The compounds presenting highest prebiotic potential were then incorporated in a rice muffin, in order to increase its commercial and nutritional value. Muffins containing $100 \%$ of sugar and $75 \%$ of sugar supplemented with prebiotics were produced and compared with a commercial rice muffin.

The main challenges of this work were (a) to confirm the in vitro prebiotic potential of marketed GOS and agave inulin and (b) to create a healthier and potentially functional pastry option for consumers who either follow a health-based lifestyle or have a special medical condition.

\section{Materials and methods}

\subsection{Materials}

Agave inulin was purchased from Entelees ${ }^{\circledR}$ (Guadalajara, Mexico) and GNC Prebiotic GOS from GNC ${ }^{\circledR}$ (Cleveland, Ohio, USA). All chemicals and media components were of analytical grade and obtained from Sigma - Aldrich Quimica S.L. (Lisboa, Portugal). Except for the prebiotics, the ingredients used for muffins preparation were purchased on a local market: Branca de Neve wheat flour from Fábricas Lusitana, S. A. (Alcains, Portugal), Ceifeira rice flour from Dacsa Atlantic, S.A. (Lisbon, Portugal), sugar from RAR, S.A. (Porto, Portugal), Vaqueiro butter from Upfield Inc. (Lisbon, Portugal), medium size eggs from Derovo, S.A. (Pombal, Portugal), Terra Nostra semi-skimmed milk from Bel Portugal, S.A. (Ribeira Grande, Açores, Portugal) and Royal baking soda from AMD Portugal, S.A. (Mora, Portugal).

\subsection{Prebiotic potential}

\subsubsection{In vitro batch culture fermentations}

Fecal samples were obtained from one healthy female donor, aged 23 years old. A voluntary informed consent was obtained from the fecal donor prior to this study. The samples were collected on site, diluted 1/ $10(\mathrm{w} / \mathrm{w})$ in anaerobic $\left(100 \% \mathrm{~N}_{2}\right)$ phosphate-buffered saline solution (PBS, $0.1 \mathrm{M}, \mathrm{pH}$ 7.0). Static batch culture fermentations and samples analysis were performed as descrived in Amorim et al. (2020a; 2020b). The nucleotide sequences were deposited in the European Nucleotide Archive (ENA) under the BioProject accession number PRJEB37799.

\subsection{Development of the traditional rice muffin}

\subsubsection{Preparation of the muffins}

Control muffins (100S) were prepared according to the original recipe of the rice muffin, containing ( $\% \mathrm{w} / \mathrm{w})$ : sugar (23.4), butter (9.4), eggs (19.1), milk (14.0), wheat flour (28.1), rice flour (5.6) and baking soda (0.5). The modified muffins were prepared based on the original recipe with a $25 \%(\mathrm{w} / \mathrm{w})$ reduction of sugar and a $0.8 \%(\mathrm{w} / \mathrm{w})$ addition of the most promising prebiotic (\% w/w): sugar (18.4), butter (9.8), eggs (20.0), milk (14.7), wheat flour (29.3), rice flour (5.9), baking soda (0.5), GOS (0.8) and GOS excipients (0.7). Butter, sugar, baking soda and the lemon flavour were mixed using a hand mixer (BHM3133, Becken, Portugal) for $5 \mathrm{~min}$. The eggs were added and mixed ( $3 \mathrm{~min}$ at speed 2) one by one. After incorporating the wheat and rice flour and when required GOS ( 1 min mixing at speed 2 ) into the batter, the milk was added, mixing $3 \mathrm{~min}$ at speed 2 . The batter was divided in $80 \mathrm{~g}$ aliquots into paper molds, and baked at $180{ }^{\circ} \mathrm{C}$ during $30 \mathrm{~min}$ in a preheated electric oven (Balay 3HB569XC, BSH, Spain). The muffins were left to cool overnight at $25{ }^{\circ} \mathrm{C}$ and at controlled atmosphere (relative humidity of $53 \pm 5 \%$ ).

\subsubsection{Physical, optical and textural properties}

The height and diameter of the muffins were measured using a digital caliper. Moisture content, $\mathrm{X} \mathrm{kg}$ water/ $\mathrm{kg}$ dry solid (d.s.), of the muffins was determined by weighing after vacuum drying at $70^{\circ} \mathrm{C}$ and $15 \mathrm{kPa}$ (AOAC, 1995). Baking mass loss, BML, was determined as described by Heo et al. (2019), using Equation (1):

$B M L=\frac{\left(w_{\text {batter }}-w_{\text {muffin }}\right) 100}{w_{\text {batter }}}$

where $\mathrm{w}_{\text {batter }}$ and $\mathrm{w}_{\text {muffin }}$ are the weights of the sample before and after baking (1 day). The color of the muffin crumbs was measured 1 day after baking, using a colorimeter (CR-400 Konica Minolta, Japan) previously calibrated, by means of six measurements on four different positions. The color parameters ( $\mathrm{L}^{*}, \mathrm{a}^{*}$ and $\left.\mathrm{b}^{*}\right)$ were accessed as described by Fariña et al. (2019). The total color difference $\left(\Delta \mathrm{E}^{*}\right)$ between samples was determined by:

$\Delta \mathrm{E}^{*}=\sqrt{\left(L^{*}-L_{r}^{*}\right)^{2}+\left(a^{*}-a_{r}^{*}\right)^{2}+\left(b^{*}-b_{r}^{*}\right)^{2}}$

where the suffix r corresponds to the reference system (Hunter \& Harold, 1987). The muffins were cut transversally and images of the cut surface of the crumb were captured. Images were analysed using the UTHSCSA ImageTool 3.0 program (ImageTool software copyright, University of Texas Health Science Center San Antonio, USA). The number of bubbles (with area above $0.008 \mathrm{~cm}^{2}$ ) was counted along with their dimensions to evaluate the average pore diameter (after the determination of the length of major and minor axes by the irregular shape of bubbles) and area. Apparent density $\left(\mathrm{g} / \mathrm{cm}^{3}\right)$ of the crumb was evaluated after cut a cylinder with a hollow punch and knife and measure its volume, $\mathrm{V}$ $\left(\mathrm{cm}^{3}\right)$, and weight, $\mathrm{w}(\mathrm{g})$. Apparent porosity, $\phi(\%)$, (bubble volume $\mathrm{x}$ 100 /sample volume) of muffin crumb was evaluated after the determination of the sample volume and the volume of the grounded sample. Specific density, $\rho_{s}\left(\mathrm{~g} / \mathrm{cm}^{3}\right)$, of the crumb was evaluated by Equation (3):

$\rho_{s}=\frac{w}{V\left(1-\frac{\varphi}{100}\right)}$

The texture profile analysis (TPA) was performed using the texture analyzer (Stable Micro System Ltd, Model TA-XT2i) apparatus. Cylindric samples of $19.90 \mathrm{~mm}$ (d) x $20 \mathrm{~mm}$ (h) were cut from the bulk of the muffins. Muffin samples were double compressed to $50 \%$ of the original height, using a flat-ended cylindrical probe $(\mathrm{P} / 25)$, a trigger force of $5 \mathrm{~g}$, pre- and test-speed of $1 \mathrm{~mm} / \mathrm{s}$ and a post-speed of $2 \mathrm{~mm} / \mathrm{s}, 10 \mathrm{~mm}$ of distance and a rest period of $5 \mathrm{~s}$ between cycles. Textural measurements were performed during 4 consecutive days for muffins stored under controlled conditions $\left(25{ }^{\circ} \mathrm{C}\right.$ and $53 \pm 5 \%$ of relative humidity). Hardness $(\mathrm{g})$, springiness $(\mathrm{cm})$, cohesiveness $(-)$, chewiness, $(\mathrm{g} \mathrm{cm})$, and resilience (-) were recorded form curves because are considered the most relevant textural parameters for muffins (Fariña et al., 2019; Goswami 

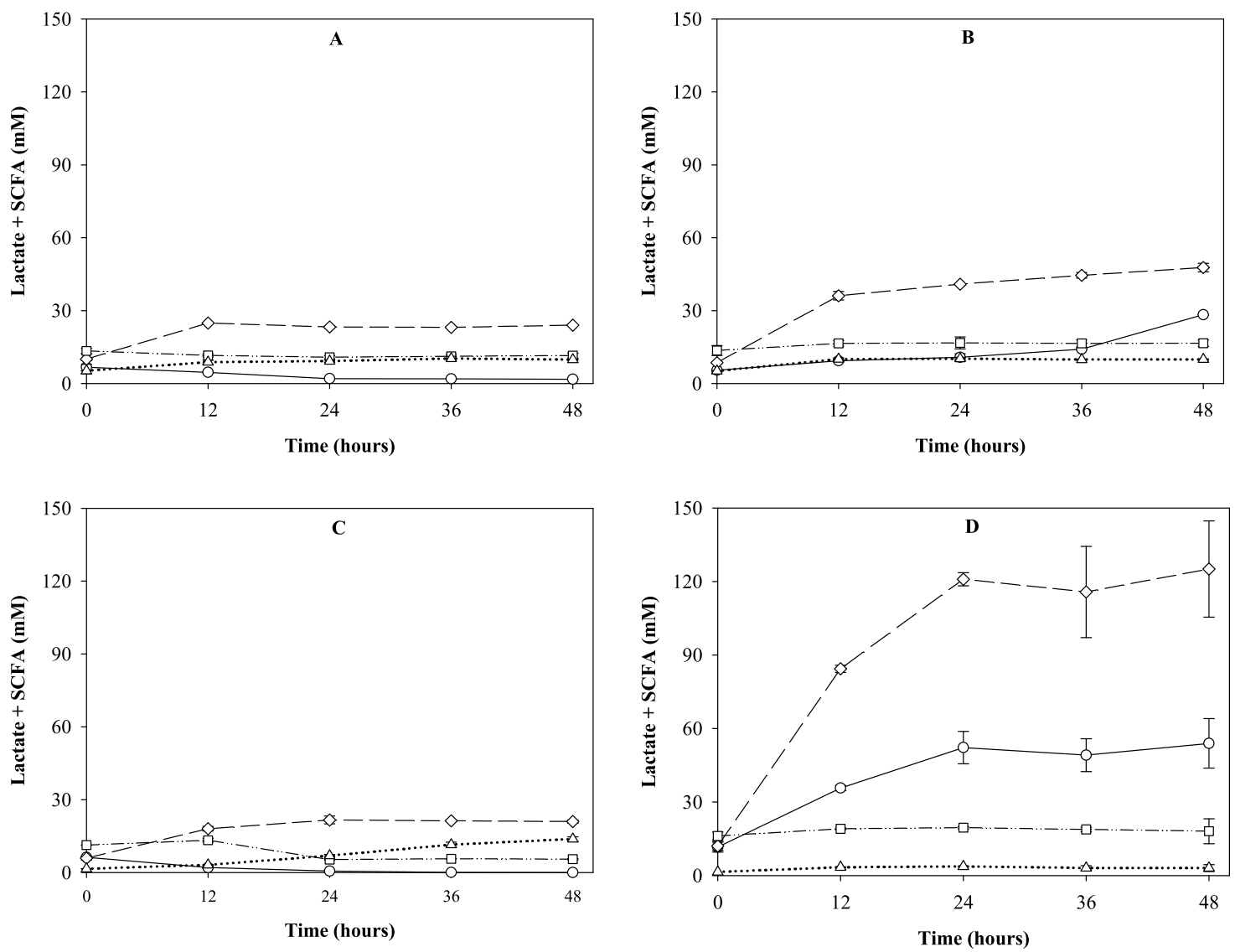

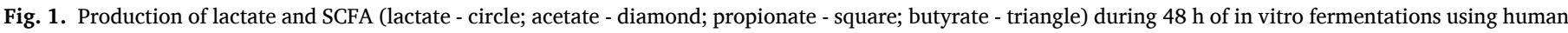

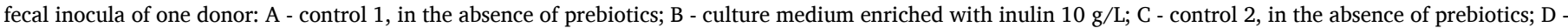
culture medium enriched with GOS $10 \mathrm{~g} / \mathrm{L}$. The results are the average of two independent fermentations \pm standard deviation.

et al., 2015). Four different muffins from each recipe were measured in duplicate. Water adsorption isotherms were determined by a static gravimetric method employing saturated salt solutions to generate a range of water activities, $\mathrm{a}_{\mathrm{w}}$, from 0.11 to 0.92 at $25{ }^{\circ} \mathrm{C}$. Triplicate samples $(1.0 \pm 0.1 \mathrm{~g})$ of pre-dried muffins at $45{ }^{\circ} \mathrm{C}$ during $12 \mathrm{~h}$ were employed ( $\mathrm{X}$ below 0.002 d.s.). At $\mathrm{a}_{\mathrm{w}}$ higher than 0.5 thymol was employed in order to avoid microbial spoilage of samples. Around 14 weeks were necessary to achieve the equilibrium. Sorption isotherms were modelled using the Oswin model (Oswin, 1946), Equation (4):

$X_{e q}=a\left(\frac{a_{w}}{1-a_{w}}\right)^{b}$

where $\mathrm{X}_{\mathrm{eq}}$ (d.s.) is the equilibrium moisture content and $\mathrm{a}$ and $\mathrm{b}$ are the fitting parameters.

\subsection{Statistical analysis}

Results are presented as mean values \pm standard deviation. The statistical significance and differences were evaluated by one-way ANOVA using the software Prism version 7.05a (GraphPad Software Inc., California, USA). Tukey test was used for post hoc comparisons. Unpaired t-test was used when required. Significant differences were considered when $\mathrm{p}<0.05$.

\section{Results and discussion}

\subsection{In vitro evaluation of the prebiotic potential}

\subsubsection{Production of lactate and short-chain fatty acids (SCFA)}

The fermentation of prebiotic oligosaccharides by gut microbiota results in the production of SCFA and lactate, a precursor of SCFA, which play an important role on human health (Gill et al., 2018). The production of lactate (that in the gut can be a precursor of SCFA), acetate, propionate and butyrate during $48 \mathrm{~h}$ of fermentation with commercial inulin and GOS is presented in Fig. 1 together with the respective controls, conducted in the absence of prebiotics.

Similar results were obtained for the controls, although representing fecal samples from different days, which suggest inter-assay reproducibility of the technique (Fig. 1). In fact, the gut microbiota of each individual is relatively stable, although varying with different factors, including age, diet, and health condition (Rinninella et al., 2019). Regarding the experiments with inulin, after $48 \mathrm{~h}$ of fermentation a significant increase in the accumulation of acetate $(24.00 \pm 0.06 \mathrm{mM}$ for control 1 and $48 \pm 2 \mathrm{mM}$ for inulin, $\mathrm{p}<0.0001)$ and lactate $(1.77 \pm$ $0.01 \mathrm{mM}$ for control 1 and $28.3 \pm 0.2 \mathrm{mM}$ for inulin, $\mathrm{p}<0.0001$ ) was observed when compared to the control fermentation (Fig. 1). While propionate showed a less statistically significant accumulation at the same time point $(12 \pm 1 \mathrm{mM}$ for control 1 and $17 \pm 1 \mathrm{mM}$ for inulin at $48 \mathrm{~h}, \mathrm{p}<0.05$ ), and butyrate revealed no significant variations relative to the control. When compared to inulin assays, the GOS fermentation led to a 2.9-fold higher relative production of lactate and SCFA at $48 \mathrm{~h}$. Additionally, the fermentations supplemented with GOS showed an increased relative production of lactate and SCFA at an early time point $(12 \mathrm{~h})$ than inulin, having reached maximum values at $24 \mathrm{~h}(571 \pm 29 \%$ 

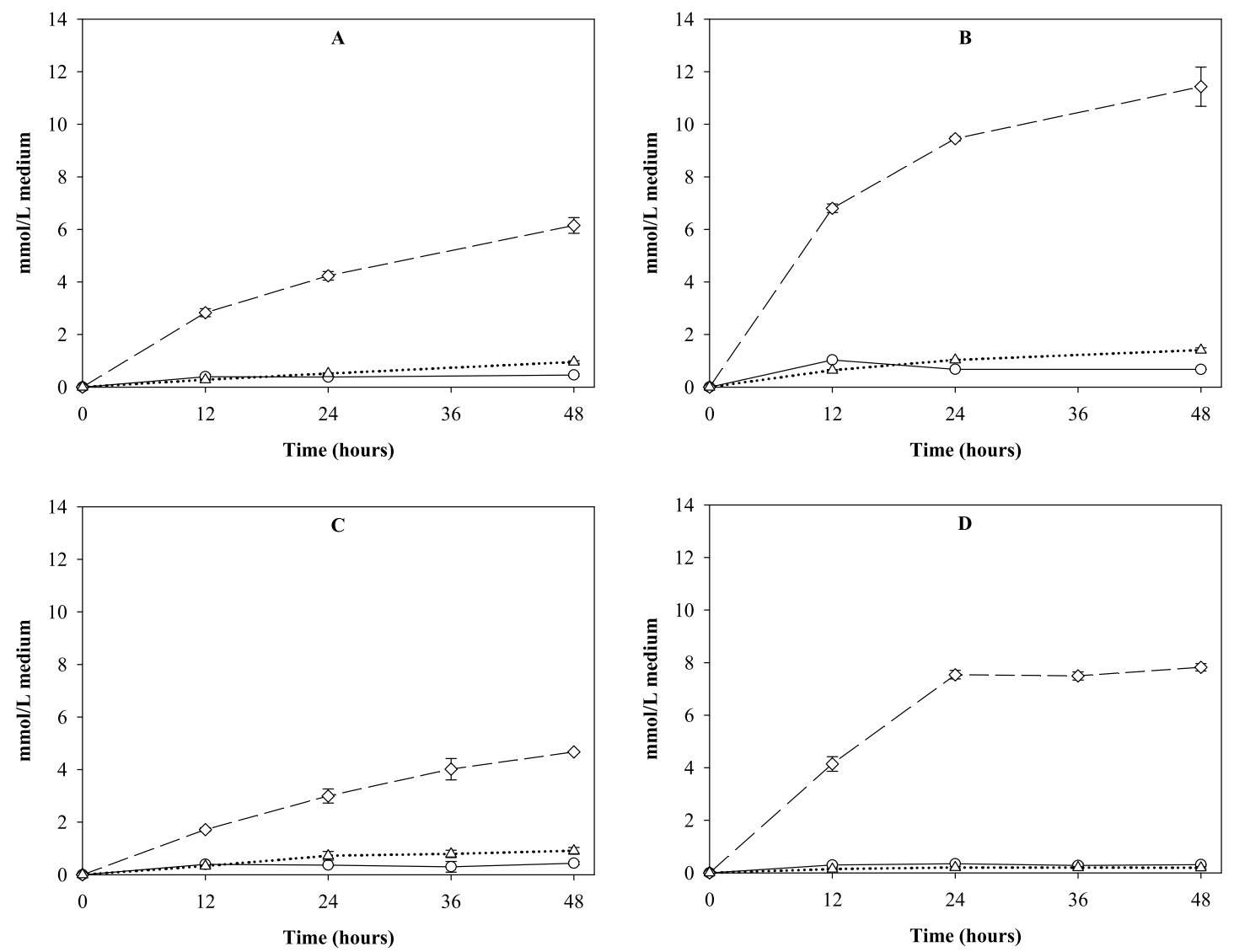

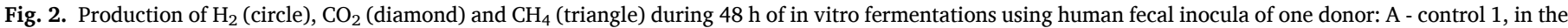

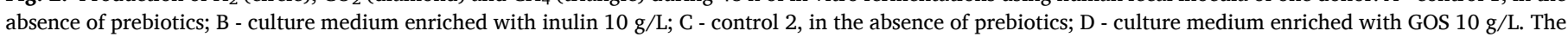
results are the average of two independent fermentations and triplicate analysis of each sample \pm standard deviation.

relative to the control). Contrariwise to inulin and GOS fermentations, the controls show a consumption of lactate leading to their absence after $48 \mathrm{~h}$, suggesting that the growth of lactate-producing bacteria is being stimulated. Overall, acetate was the main SCFA produced, followed by the organic acid lactate, with propionate and butyrate being the least produced. Lactate and acetate production were significantly higher in experiments using GOS rather than inulin.

Rycroft et al. (2001) reported comparable results with increased production of acetate, propionate and lactate resulting from GOS supplementation when compared to inulin, both added at a concentration of $10 \mathrm{~g} / \mathrm{L}$. It is comprehensible that acetate was the most produced metabolite since its production pathways are present in many bacterial groups, while lactate, propionate and butyrate production pathways exhibit a more conserved distribution (Louis et al., 2007; Morrison \& Preston, 2016). On the other hand, it is also known that inulin and GOS promote the growth of Bifidobacteria which are known to be non-butyrogenic bacteria (Gibson et al., 2017; Louis et al., 2007). The substrate concentration of $10 \mathrm{~g} / \mathrm{L}$ is a widely used in in vitro models with supplementation of different prebiotics, including inulin and GOS (Amorim et al., 2020a, 2020b; Kanjan \& Hongpattarakere, 2017; Pompei et al., 2008; Rodriguez-Colinas et al., 2013; Rycroft et al., 2001), therefore the results from this study could be easily compared with the literature. In addition, it is important to mention that this substrate concentration promoted significant changes on the metabolite production profiles when compared to the blank, thus avoiding technical limitations regarding sensitivity issues variations. However, after this first assessment of the prebiotic potential, the authors consider very relevant to determine the influence of the substrate concentration on the production profiles (i.e. study a wide range of concentrations) in a future work.

\subsubsection{Production of ammonia and final $\mathrm{pH}$ variation}

The accumulation of SCFA decreases the medium $\mathrm{pH}$, promoting the reduction of pathogenic bacteria and allowing the increase of the population of beneficial bacteria. The change in the microbial population may lead to alterations in the metabolic pathways, such as the prevalence of saccharolytic fermentation over proteolytic fermentation, which in turn causes a reduction of ammonia production. Ammonia contributes to foul fecal odour and, being a toxic compound, can lead to colon carcinogenesis (Louis et al., 2007; Power et al., 2014). The final $\mathrm{pH}$ and ammonia concentration after $48 \mathrm{~h}$ of fermentation were determined in the control and the supplemented fermentations with prebiotics (Supplementary material, Table S1). A similar decrease in the $\mathrm{pH}$ value was observed due to inulin $(\Delta \mathrm{pH}=3.32)$ and GOS fermentations $(\Delta \mathrm{pH}=3.26)$. However, GOS resulted in a higher decrease in the ammonia concentration $(398 \pm 4 \mathrm{mg} / \mathrm{L}$ at $48 \mathrm{~h}$ ), with a 5.4-fold reduction compared to the control fermentation (Supplementary material, Table S1). Pompei et al. (2008) reported similar results with a significant decrease of $\mathrm{pH}$ value in in vitro static batch fermentations of fecal microbiota supplemented with oligofructose, a well-established prebiotic, in comparison to the control fermentations. These authors also described a lower concentration of ammonia as a result of oligosaccharide supplementation.

The preference for saccharolytic fermentation and acidic $\mathrm{pH}$ are usual consequences associated to the in vitro models as used in this study, where only the distal colon conditions are mimicked and the medium $\mathrm{pH}$ is not controlled throughout the fermentation. Thus, the in vitro models are regarded as a semi-representation of the in vivo models due to their intrinsic limitations. Contrary to the in vivo models, the SCFA are not absorved and the different intestinal sections are not fully replicated. On the other hand, an in vitro model is a better approach to 


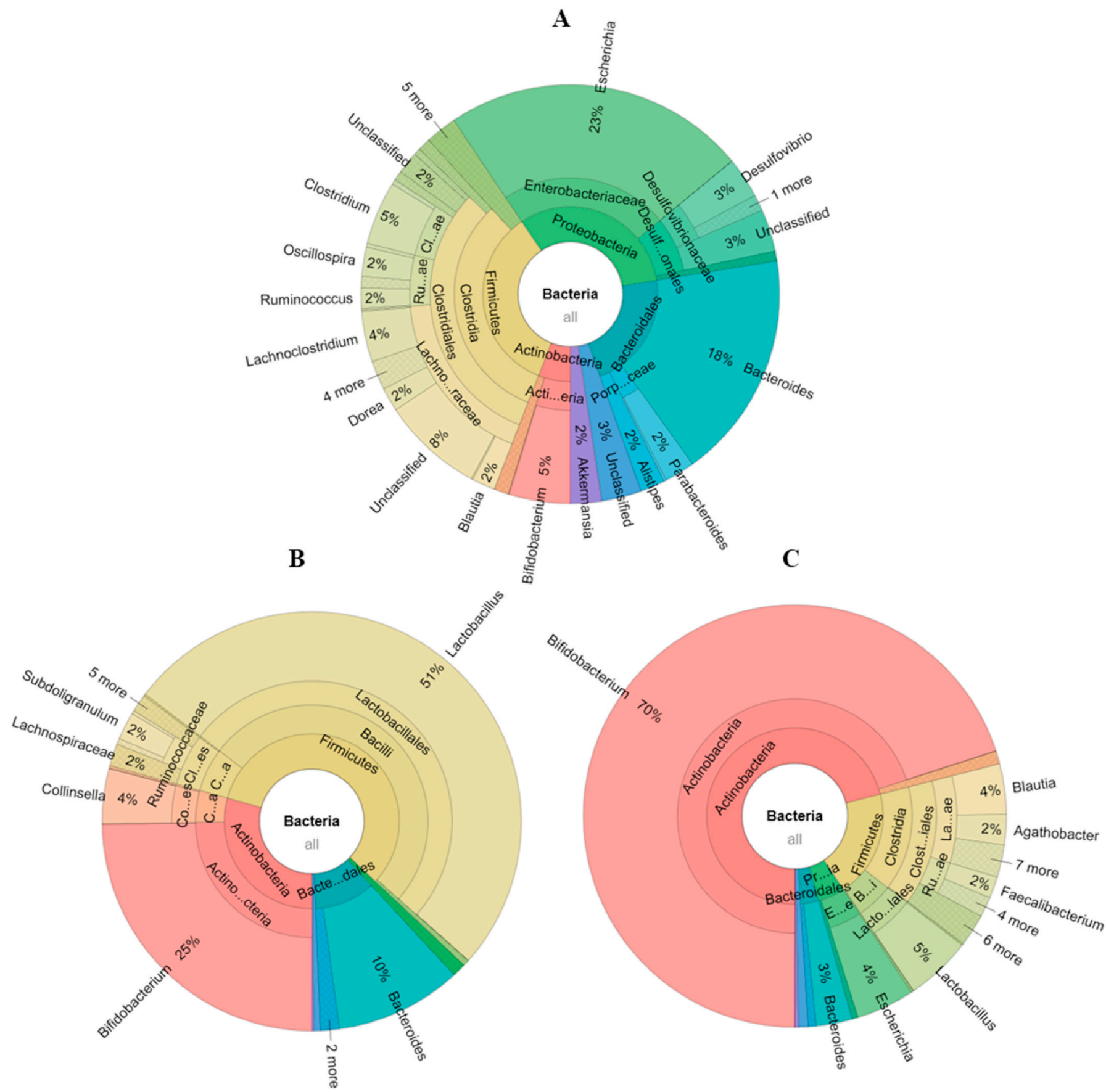

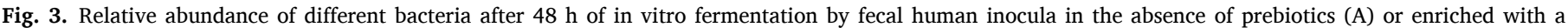
prebiotic solution of commercial agave inulin (B) or GOS (C) at $10 \mathrm{~g} / \mathrm{L}$.

evaluate the kinetics of colonic fermentation and to screen the prebiotic potential of different compounds (Payne et al., 2012).

\subsubsection{Gas production}

The fermentation of oligosaccharides by fecal human microbiota affects the production of gases such as carbon dioxide $\left(\mathrm{CO}_{2}\right)$, hydrogen $\left(\mathrm{H}_{2}\right)$ and methane $\left(\mathrm{CH}_{4}\right) . \mathrm{CH}_{4}$ is only produced in a small portion of the population, who harbour methanogenic bacteria in their gut microbiota (Ghoddusi et al., 2007). Fig. 2 presents the gas production profiles during the $48 \mathrm{~h}$ of fermentation supplemented with inulin or GOS, and respective controls. As it occurred for the SCFA, the controls exhibited similar production profiles although carried out in different occasions, with different fecal samples from the same donor. For both compounds, higher concentrations of $\mathrm{CO}_{2}$ were obtained when comparing to the control ( $\mathrm{p}<0.0001$ ), presenting maximum concentrations at $48 \mathrm{~h}$ for inulin $(11 \pm 1 \mathrm{mmol} / \mathrm{L})$ and at $24 \mathrm{~h}$ for GOS $(7.5 \pm 0.1 \mathrm{mmol} / \mathrm{L})$, corresponding to a respective 1.9 - and 2.5 -fold increase relative to the control.

Regarding the production of $\mathrm{CH}_{4}$, no significant differences were observed between control and inulin fermentation, contrary to the GOS fermentation. Interestingly, the GOS supplementation led to a decrease of 4.7-fold in the production of $\mathrm{CH}_{4}$ when compared to the control. The composition (\%) of the gaseous phase, in terms of $\mathrm{CO}_{2}, \mathrm{H}_{2}$ and $\mathrm{CH}_{4}$, after $48 \mathrm{~h}$ of fermentation highlights the evidence that GOS addition led to the lower percentage of $\mathrm{CH}_{4}$. (Supplementary material, Fig. S1). Furthermore, the supplementation with GOS resulted in no significant 
alterations in $\mathrm{CH}_{4}$ concentration from $12 \mathrm{~h}$ to $48 \mathrm{~h}(0.14 \pm 0.02 \mathrm{mmol} / \mathrm{L}$ and $0.19 \pm 0.04 \mathrm{mmol} / \mathrm{L}$, respectively), while in the control increased amounts of $\mathrm{CH}_{4}$ were produced $(0.33 \pm 0.04 \mathrm{mmol} / \mathrm{L}$ at $12 \mathrm{~h}$ and $0.91 \pm$ $0.13 \mathrm{mmol} / \mathrm{L}$ at $48 \mathrm{~h}, \mathrm{p}<0.0001$ ) (Fig. 2). Elevated $\mathrm{CH}_{4}$ production has been associated with obesity, colorectal cancer, irritable bowel syndrome and chronic constipation. In fact, said gas has been shown to reduce intestinal transit time by $59 \%$ (Gaci et al., 2014; Lurie-Weinberger \& Gophna, 2015). Therefore, further studies on the ability of GOS to reduce methane production should be carried out. The highest total gas production occurred as a result of inulin supplementation, while GOS supplementation led to a lower overall gas production. Similar results were reported by Hernot et al. (2009) and Rycroft et al. (2001) who compared the effect of different oligosaccharides. Hernot et al. (2009) after evaluating the gas production profile of fructans with different chain lengths and DP and other oligosaccharides, such as GOS, concluded that due to its branched structure, agave inulin was more rapidly fermented yielding the highest amounts of gas, while GOS yielded more moderate gas production. Similarly, in the results reported by Rycroft et al. (2001), the gas production profiles obtained revealed that fructooligosaccharides (FOS) and inulin produced the highest amounts of total gas, while GOS resulted in the lowest production. GOS are considered more prone to increase the number of Bifidobacteria than inulin, which could explain the differences herein found in the gas production, since these bacteria usually generate lower gas volumes.

\subsubsection{Microbiota analysis}

Taking into account the results herein gathered, GOS presented the highest prebiotic potential and therefore, were selected to be incorporated in the rice muffins. The supplementation with inulin or GOS resulted in a distinct modulation of the gut microbiota after $48 \mathrm{~h}$ of in vitro fermentation (Fig. 3). Despite the fermentation of both prebiotics increased the relative abundance of members of Lactobacillaceae and Bifidobacteriaceae families, inulin presented a higher selectivity towards Lactobacillaceae strains ( $51 \pm 1 \%$ of all), while GOS showed to be more efficient in stimulating the growth of Bifidobacteriaceae strains ( $65 \pm 7 \%$ of all). Members of these families, such as Lactobacillus acidophilus, Limosilactobacillus fermentum, Lacticaseibacillus paracasei, Lacticaseibacillus rhamnosus, (formerly named Lactobacillus fermentum, Lactobacillus paracasei and Lactobacillus rhamnosus, respectively, emend. Zheng et al., 2020), Bifidobacterium longum and Bifidobacterium bifidum, are widely commercialized probiotic linked to several health benefits (Fenster et al., 2019; Silvi et al., 2003; Zheng et al., 2020). They are able to metabolize complex carbohydrates, producing mainly acetate and lactate and releasing $\mathrm{CO}_{2}$ (Gibson et al., 2017), which corroborates the results previously presented. Additionally, the fermentation of inulin and GOS led to a significant reduction of the relative abundance of Proteobacteria, including Enterobacteriaceae strains, namely Escherichia coli (108 \pm 51 - and $38 \pm 1$ - fold, respectively) (Supplementary material, Table S2). The reduction of the relative abundance of species belonging to Lachnospiraceae family (e.g. Blautia sp.) $(6 \pm 1$ - fold for inulin and $2.3 \pm 0.3$ - fold for GOS) and the high reduction of Erysipelotrichaceae strains ( $51 \pm 48$ - fold for inulin $23 \pm 8$ fold for GOS) was also shown for both prebiotics. The abundance of species from Enterobacteriaceae, Lachnospiraceae and Erysipelotrichaceae on gut microbiota is associated wtih gastrointestinal disorders, including inflammatory bowel disease (Rizzatti et al., 2017), visceral fat accumulation (Ozato et al., 2019) and colorectal cancer (Dinh et al., 2014). The presence of methanogenic archaea was also reduced by both prebiotics, in particular by GOS which led to the null values of its relative abundance, corroborating the decrease of $\mathrm{CH}_{4}$ production previously observed (Supplementary material, Table S2). Taking into account the results herein gathered, GOS presented the highest prebiotic potential and therefore, were selected to be incorporated in the rice muffins. Further investigations should be performed to determine if the commercial GOS could be used as vehicle matrices to
Table 1

Physical properties and texture profile analysis of the studied muffins one day after their production: 100 (rice muffin with $100 \%$ of sugar); $75 \mathrm{GOS}$ ( $75 \%$ of sugar and $0.8 \%$ GOS; and Commercial (commercial rice muffin). A different superscript letter in the same row indicates statistical difference $(p<0.05)$.

\begin{tabular}{|c|c|c|c|}
\hline & $100 \mathrm{~S}$ & $75 \mathrm{GOS}$ & Commercial \\
\hline Height (cm) & $6.3 \pm 0.4^{\mathrm{a}}$ & $5.6 \pm 0.1^{b}$ & $7.6 \pm 0.5^{c}$ \\
\hline Weight $(g)$ & $74 \pm 1^{\mathrm{a}}$ & $75 \pm 1^{\mathrm{a}}$ & $93 \pm 8^{\mathrm{b}}$ \\
\hline Diameter $(\mathrm{cm})$ & $6.0 \pm 0.1^{\mathrm{a}}$ & $6.0 \pm 0.1^{\mathrm{a}}$ & $6.0 \pm 0.2^{\mathrm{a}}$ \\
\hline Volume $\left(\mathrm{cm}^{3}\right)$ & $\begin{array}{l}178.9 \pm \\
0.4^{\mathrm{a}}\end{array}$ & $158.9 \pm 0.2^{\mathrm{a}}$ & $212 \pm 15^{\mathrm{b}}$ \\
\hline Baking loss (\%) & $9.0 \pm 1.0^{\mathrm{a}}$ & $9.1 \pm 0.3^{\mathrm{a}}$ & - \\
\hline Apparent density of crumb $\left(\mathrm{g} / \mathrm{cm}^{3}\right)$ & $\begin{array}{l}0.28 \pm \\
0.01^{\mathrm{a}}\end{array}$ & $0.36 \pm 0.01^{\mathrm{b}}$ & $0.33 \pm 0.03^{\mathrm{ab}}$ \\
\hline Moisture content (d.s.) & $\begin{array}{l}0.35 \pm \\
0.03^{\mathrm{a}}\end{array}$ & $0.38 \pm 0.02^{\mathrm{a}}$ & $0.49 \pm 0.07^{\mathrm{b}}$ \\
\hline Apparent porosity of crumb (\%) & $\begin{array}{l}74.3 \pm \\
0.1^{\mathrm{a}}\end{array}$ & $68 \pm 1^{b}$ & $68 \pm 2^{b}$ \\
\hline Number of bubbles ${ }^{\mathrm{a}} / \mathrm{cm}^{2}$ & $0.7 \pm 0.1^{\mathrm{a}}$ & $2.2 \pm 0.1^{\mathrm{b}}$ & $1.8 \pm 0.2^{\mathrm{b}}$ \\
\hline Bubble area $\left(\mathrm{cm}^{2}\right)$ & $\begin{array}{l}0.07 \pm \\
0.03^{\mathrm{ab}}\end{array}$ & $0.04 \pm 0.02^{\mathrm{a}}$ & $0.06 \pm 0.06^{\mathrm{b}}$ \\
\hline Bubble diameter $(\mathrm{cm})$ & $\begin{array}{l}0.29 \pm \\
0.08^{\mathrm{a}}\end{array}$ & $0.23 \pm 0.06^{\mathrm{a}}$ & $0.2 \pm 0.1^{\mathrm{a}}$ \\
\hline Specific density of crumb $\left(\mathrm{g} / \mathrm{cm}^{3}\right)$ & $\begin{array}{l}1.60 \pm \\
0.01^{\mathrm{a}}\end{array}$ & $1.49 \pm 0.01^{\mathrm{b}}$ & $1.35 \pm 0.01^{\mathrm{c}}$ \\
\hline \multicolumn{4}{|l|}{ Color } \\
\hline$L^{*}$ & $66 \pm 1^{\mathrm{a}}$ & $68 \pm 2^{\mathrm{a}}$ & $57 \pm 2^{\mathrm{b}}$ \\
\hline$a^{*}$ & $\begin{array}{l}-2.2 \pm \\
0.1^{\mathrm{a}}\end{array}$ & $-2.5 \pm 0.1^{\mathrm{a}}$ & $-1.4 \pm 0.3^{b}$ \\
\hline $\mathrm{b}^{*}$ & $\begin{array}{l}23.3 \pm \\
0.3^{\mathrm{a}}\end{array}$ & $27 \pm 1^{\mathrm{b}}$ & $26 \pm 1^{\mathrm{b}}$ \\
\hline$\Delta \mathrm{E}^{*}$ & $9 \pm 1^{\mathrm{a}}$ & $11 \pm 2^{\mathrm{a}}$ & - \\
\hline Hardness (g) & $205 \pm 47^{\mathrm{a}}$ & $372 \pm 49^{\mathrm{b}}$ & $209 \pm 35^{\mathrm{a}}$ \\
\hline Springiness $(\mathrm{cm})$ & $\begin{array}{l}0.89 \pm \\
0.02^{\mathrm{a}}\end{array}$ & $0.86 \pm 0.01^{b}$ & $0.82 \pm 0.02^{c}$ \\
\hline Cohesiveness (-) & $\begin{array}{l}0.70 \pm \\
0.02^{\mathrm{a}}\end{array}$ & $0.60 \pm 0.04^{b}$ & $0.39 \pm 0.03^{c}$ \\
\hline Resilience (-) & $\begin{array}{l}0.28 \pm \\
0.01^{\mathrm{a}}\end{array}$ & $0.23 \pm 0.02^{\mathrm{b}}$ & $0.13 \pm 0.01^{c}$ \\
\hline Chewiness ( $\mathrm{g} \mathrm{cm}$ ) & $129 \pm 29^{a}$ & $190 \pm 19^{b}$ & $67 \pm 13^{c}$ \\
\hline
\end{tabular}

${ }^{\mathrm{a}}$ Pores with area higher than $0.008 \mathrm{~cm}^{2}$.

increase the survival rate of specific probiotic strains when tested on simulated gastrointestinal transit conditions (Pérez-Ramos et al., 2017). The most efficient combination of prebiotic and probiotic could be used afterwards to develop a synbiotic rice muffin.

\subsection{Physical, structural and textural properties of rice muffins}

Given the relevance of the GOS fermentation results, it would be interesting to transpose their potential beneficial effects to a highly consumed food product with a low level of innovation, such as the rice muffin, in order to increase its commercial and nutritional value. In this study, rice muffins were produced containing $100 \%$ of the sugar amount present in the original recipe (100S), $75 \%$ of sugar supplemented with $0.8 \%$ GOS (75GOS), and were then compared with a commercial muffin (Commercial). The concentration of $0.8 \%$ of GOS was established attending to the official recommended daily dose of the product $(1.37 \mathrm{~g} /$ day) and assuming that a maximum of 2 cakes/day could be eaten by one person. Concentrations of GOS higher than the recommended daily dose could result in the occurrence of undesired secondary effects, including flatulence and diarrhoea. Thus, as a first approach, the authors selected a conservative concentration of GOS to ensure no repercussions for the consumers' well-being. On the other hand, the $25 \%$ sugar reduction was chosen based on the outputs of a preliminary sensory test with 50 subjects (data not shown). As expected, muffins with $25 \%$ sugar reduction were more well accepted in terms of their organoleptic properties than with $50 \%$ sugar reduction, since sugar plays an important role in the texture, flavour and moisture of the muffins. The physical characterization of the baked rice muffins are summarized in Table 1. Baking loss values are comparable to previous results corresponding to 

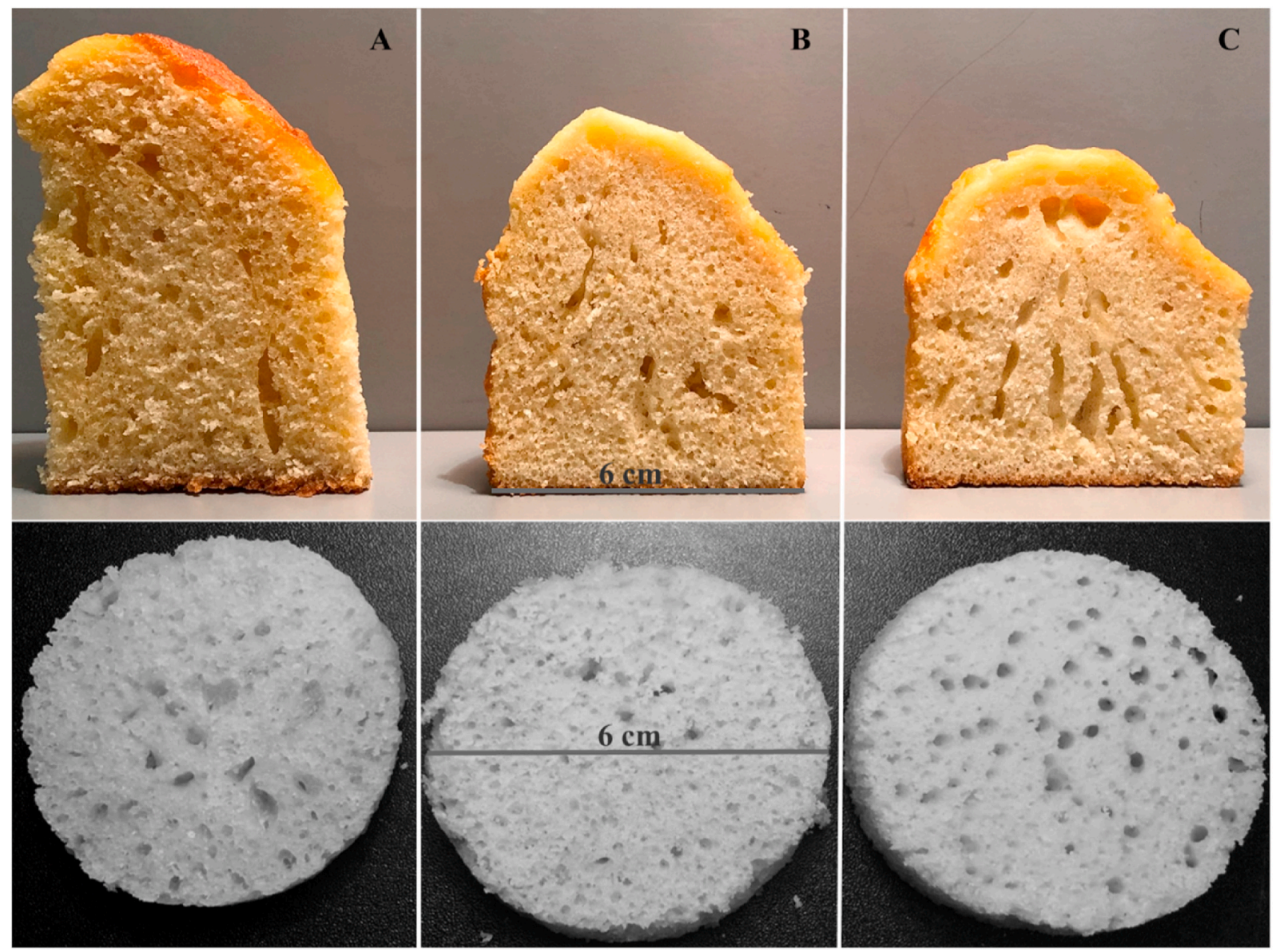

Fig. 4. Vertical and transversal sections of the studied muffins one day after their production: A) Commercial rice muffin (Commercial); B) 100\% of sugar (100S) and C) $75 \%$ of sugar and $0.8 \%$ GOS (75GOS). Note that when "Lactobacillus" is referred in this figure, refers to the formerly Lacobacillus genus, before the emended description by Zheng et al. (2020).

other muffins (Goswami et al., 2015) and no significant differences between homemade samples were observed. Regarding color parameters of the muffin crumb, only relevant differences were found in $L^{*}$ parameter for the commercial sample. Possibly, the use of higher temperatures during baking promoting Maillard reactions could explain this difference (Shevkani \& Singh, 2014). Minor colour differences between $100 \mathrm{~S}$ and 75GOS $\left(\Delta \mathrm{E}^{*}<3\right)$ can be considered (Goswami et al., 2015). The 75GOS sample showed a lower volume than the other samples. In fact, the sucrose content is relevant for the development of desired structural characteristics as it improves the starch gelatinization and protein denaturizing temperatures, thus increasing the samples volume (Hoseney, 1994). Muffins are porous food materials due to the presence of many air bubbles, Fig. 4.

The bubbles are produced during the mixing process and grow during baking. Starch gelatinization and protein denaturizing set the structure during baking and is completed with the formation of starch gel during cooling. The muffin with prebiotic exhibited a larger number of bubbles with smaller size than the other tested muffins. Nevertheless, the vertical section shows the existence of some tunnels at the centre of the muffin (Fig. 4C), which evidences that the capacity of starch and protein structure to retain the air bubbles adequately was reduced and some collapse took place (Heo et al., 2019). Despite these differences found for the porous structure, the apparent porosity of crumb (consequently, also the apparent density) of the tested muffins varied in a narrow range (from 68 to $74.3 \%$ ). Finally, specific density values are in the range of other muffins prepared with amaranth and buckwheat flours (Dizlek, 2015). A linear relationship $\left(r^{2}>0.99\right)$ between specific volume and moisture content was established.
Results are in the range of other muffins made from other flours and different additives (Alvarez et al., 2017; Chung et al., 2010). Commercial and $100 \mathrm{~S}$ muffins showed similar hardness and springiness. The muffin recipe with higher starch and protein content in its formulation (75GOS) showed a higher hardness and thus, higher chewiness values (and higher apparent density). Also, the high moisture content of the 75GOS sample could be explained by the water retention capacity of starch and protein. Springiness is a desirable muffin property related to elastic characteristics (Shevkani \& Singh, 2014). This property varied in a narrow range for the tested crumb muffins (from 0.82 up to 0.89 with significant differences between samples) and it is similar to the reported values for other muffins, suggesting an adequate acceptability of the product by the consumer (Sanz et al., 2009). No relationship was found between air bubbles characteristics or specific volume or hardness and, it seems that the developed protein network and starch gel jointly provide a muffin structure with similar elasticity for the tested samples. Despite the lower porosity of the 75GOS muffin comparing to the $100 \mathrm{~S}$ muffin, the springiness value could be due to the formation of tunnels (Chung et al., 2010). Cohesiveness measures the strength of internal bonds in the muffin and, resilience measures how well a product fights to regain its original position (Trinh \& Glasgow, 2012, pp. 23-26). Usually, linear correlations between both parameters can be established as in this case $\left(r^{2}=0.999\right)$. In parallel, both parameters increased linearly $\left(r^{2}>0.98\right)$ with increasing specific density of the tested muffins.

Texture of the samples was determined during four days after preparation. Parameters determined at day 1 (Table 1) were used as reference and relative values of every parameter were calculated. Moisture content of samples varied slightly during these four days with 

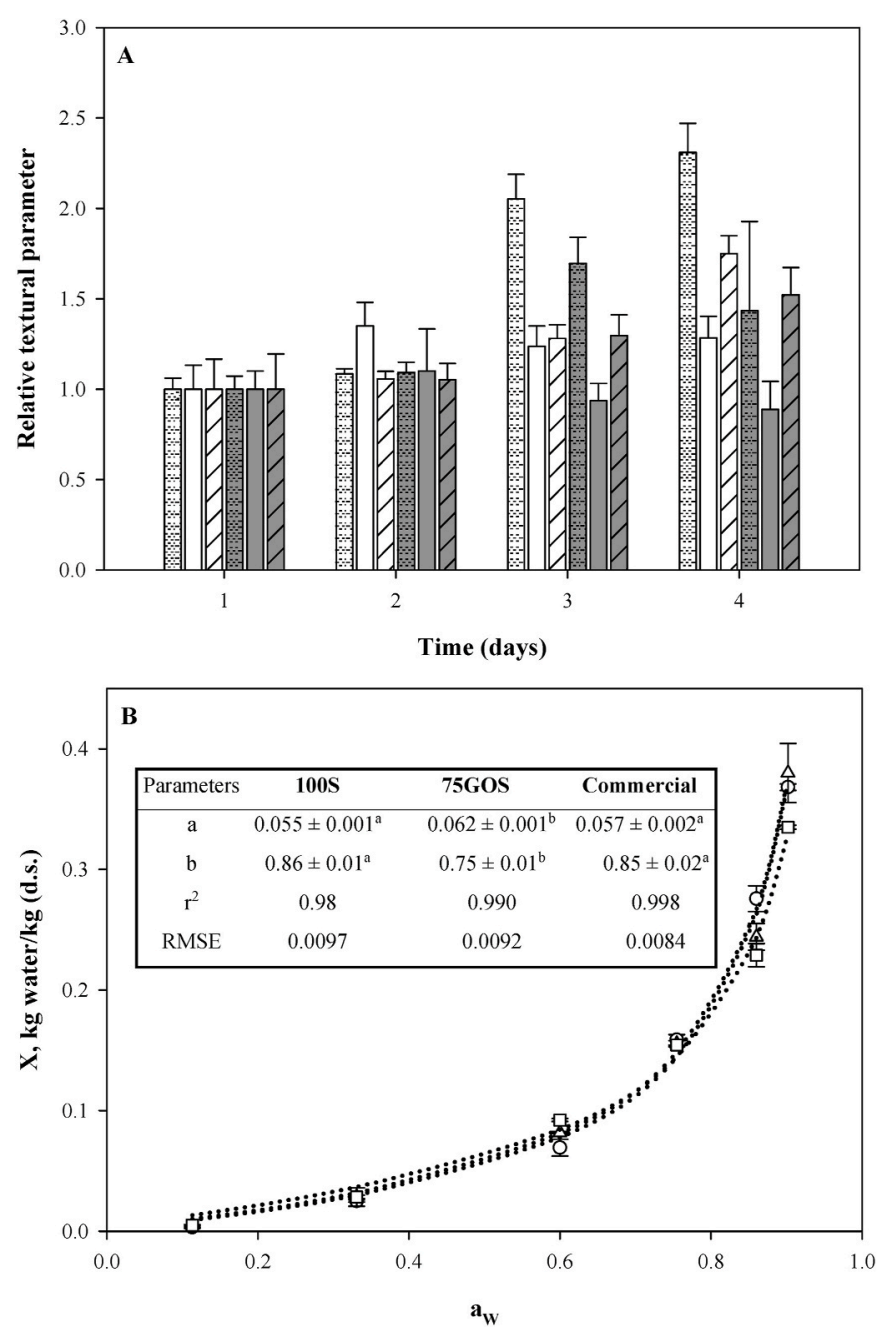

Fig. 5. Characterization of the rice cakes: A - Evolution of hardness (white) and chewiness (dark grey) during time for the rice cake with $100 \%$ of sugar (short dash), rice cake with $75 \%$ of sugar and $0.8 \%$ GOS (no fill) and commercial rice cake (long dash); B - Water adsorption isotherms obtained at $25{ }^{\circ} \mathrm{C}$ for the rice cake with $100 \%$ of sugar (triangle), rice cake with $75 \%$ of sugar and $0.8 \%$ GOS (square) and commercial rice cake (circle). Dashed lines corresponding to Oswin model (Equation (4)) fittings with the respective parameters presented in the insert (different superscript letter in the same row indicates statistical difference $(\mathrm{p}<0.05)$ ). RMSE stands for root mean square error.

an increase of $2 \pm 1$ d.s. Relative springiness continuously decreased over a narrow range (up to $0.95 \pm 0.01$ for $75 \mathrm{GOS}$ sample); relative cohesiveness also decreased but more markedly (up to 0.7 for $75 \mathrm{GOS}$ sample); and relative resilience remained constant for the Commercial sample and dramatically decreased for homemade muffins (up to $0.7 \pm$ 0.1 for 75 GOS sample). Fig. 5A shows, as an example, the evolution of hardness and chewiness during storage time. As expected, the relative hardness increased in all samples. In fact, 100S muffin after 3 days and Commercial muffin after 4 days showed values above two. Relative chewiness showed for both muffins the same trend. Relative hardness of the 75GOS sample increased slightly (up to $1.3 \pm 0.1$ ) and relative chewiness decreased (up to $0.9 \pm 0.1$ ). These last trends are interesting because initial values for both parameters were high. Nevertheless, the texture profile analysis (TPA) results with time clearly suggest that the product should conveniently be consumed during the first two days after baking.

Fig. 5B shows the water adsorption isotherms at $25{ }^{\circ} \mathrm{C}$ of the tested muffins. In all cases, the equilibrium moisture content values increase (up to 0.37 d.s.) with the increase of water activity. Therefore, water adsorption isotherms can be classified as Type III according to Brunauer's classification (Brunauer et al., 1940). Slight differences can be observed between Commercial and 100S samples, thus confirming that these samples were prepared with a similar formulation. At high water activities (above 0.8), the 75GOS sample showed lower equilibrium moisture content than the other samples due to its low sugar content (Moreira et al., 2009). The Oswin model is one of the most used models for starchy products (Brett et al., 2009). Fig. 5B shows the fitting parameters of the Oswin model for the tested muffins and the statistical parameters are indicative of the goodness of the fittings (coefficient of determination, $r^{2}>0.98$ and root mean square error, RMSE $<0.0097$ ). The water sorption isotherms indicate that the tested samples can be considered perishable food materials since they have high water activities (around 0.9). The storage of these products under a standard relative humidity (45\%) would give as result, if no spoilage takes place, a very dried product (around 0.046 d.s.). Therefore, hygroscopic and textural results allow to further conclude that these products must be consumed in a short period of time (one or two days) if additional preservatives or packaging are not used.

\section{Conclusions}

When compared with inulin, the fermentation of GOS resulted in the highest production of short-chain fatty acids, bifidogenic effect and $\mathrm{pH}$, ammonia and $\mathrm{CH}_{4}$ reduction. The GOS fermentation results confirmed their high prebiotic potential, which was further exploited into a widely consumed food product with a low level of innovation, such as the rice muffin, in order to increase its commercial and nutritional value. A rice muffin was developed containing $25 \%$ less sugar than the original recipe and $0.8 \%$ of GOS. The reduction of sugar content modified the air bubbles characteristics (size and population with a greater number of tunnels) and hardness and chewiness increased. Nevertheless, very relevant textural parameters were obtained for the new-formula rice muffins, like springiness and resilience, and these were considered acceptable and comparable to those reported in the literature for commercial muffins, thus anticipating a good consumer acceptance. Overall, the results gathered in this study comprise an important contribution for the development of potential healthier and functional pastry options designed for consumers who either follow a health-based lifestyle or have a special medical condition.

\section{Author contribution statements}

Cláudia Amorim: Funding acquisition, Investigation, Methodology, Writing - original draft Beatriz B. Cardoso: Investigation, Methodology Sara C. Silvério: Investigation, Methodology, Writing - review \& editing Jessica C. Silva: Investigation, Methodology Joana I. Alves: Investigation, Methodology, Writing - review \& editing Maria Alcina Pereira: Funding acquisition, Resources, Validation, Writing - review \& editing Ramón Moreira: Funding acquisition, Resources, Validation, Writing - review \& editing Lígia R. Rodrigues: Funding acquisition, Resources, Validation, Supervision, Writing - review \& editing, Project administration.

\section{Declaration of competing interest}

The authors confirm that they have no conflicts of interest with respect to the work described in this manuscript.

\section{Acknowledgments}

This study was supported by the Portuguese Foundation for Science and Technology (FCT) under the scope of the strategic funding of UIDB/ 04469/2020 unit and BioTecNorte operation (NORTE-01-0145-FEDER000004) funded by the European Regional Development Fund under the scope of Norte2020 - Programa Operacional Regional do Norte; 
COMPETE 2020 (POCI-01-0145-FEDER-006684), FoSynBio (POCI-010145-FEDER-029549) and NewFood (NORTE-01-0246-FEDER000043). CA and BBC acknowledge their grants (UMINHO/BPD/4/2019 and SFRH/BD/132324/2017) from FCT. RM acknowledges the financial support to the Xunta de Galicia and FEDER by the project (EDD431B 2019/01).

\section{Appendix A. Supplementary data}

Supplementary data to this article can be found online at https://doi. org/10.1016/j.fbio.2020.100858.

\section{References}

Ahmed, W., \& Rashid, S. (2019). Functional and therapeutic potential of inulin: A comprehensive review. Critical Reviews in Food Science and Nutrition, 59(1), 1-13.

Ahuja, K., \& Mamtani, K. (2019). Prebiotics Market size to exceed \$7, 2bn by 2024. Internet: https://www.gminsights.com/pressrelease/prebiotics-market-size. (Accessed September 2020).

Alvarez, M. D., Herranz, B., Jiménez, M. J., \& Canet, W. (2017). End-product quality characteristics and consumer response of chickpea flour-based gluten-free muffins containing corn starch and egg white. Journal of Texture Studies, 48(6), 550-561.

Amorim, C., Silvério, S. C., Cardoso, B. B., Alves, J. I., Pereira, M. A., \& Rodrigues, L. R. (2020a). In vitro fermentation of raffinose to unravel its potential as prebiotic ingredient. LWT - Food Science and Technology, 126, 109322

Amorim, C., Silvério, S. C., Cardoso, B. B., Alves, J. I., Pereira, M. A., \& Rodrigues, L. R. (2020b). In vitro assessment of prebiotic properties of xylooligosaccharides produced by Bacillus subtilis 3610. Carbohydrate Polymers, 229, 115460.

Amorim, C., Silvério, S. C., Prather, K. L. J., \& Rodrigues, L. R. (2019). From lignocellulosic residues to market: Production and commercial potential of xylooligosaccharides. Biotechnology Advances, 37, 107397.

AOAC. (1995). Official methods of analysis of AOAC International. Washington USA: Association of Official Analytical Chemists, Article 0935584544.

Brett, B., Figueroa, M., Sandoval, A. J., Barreiro, J. A., \& Müller, A. J. (2009). Moisture sorption characteristics of starchy products: Oat flour and rice flour. Food Biophysics, 4(3), 151-157.

Brunauer, S., Deming, L. S., Deming, W. E., \& Teller, E. (1940). On a theory of the van der Waals adsorption of gases. Journal of the American Chemical Society, 62(7), 1723-1732.

Chung, H.-J., Lee, S.-E., Han, J.-A., \& Lim, S.-T. (2010). Physical properties of dry-heated octenyl succinylated waxy corn starches and its application in fat-reduced muffin. Journal of Cereal Science, 52(3), 496-501.

Date, Y., Nakanishi, Y., Fukuda, S., Nuijima, Y., Kato, T., Umehara, M., Ohno, H., \& Kikuchi, J. (2014). In vitro evaluation method for screening of candidate prebiotic foods. Food Chemistry, 152, 251-260.

Devaraj, R. D., Jeepipalli, S. P. K., \& Xu, B. (2020). Phytochemistry and health promoting effects of Job's tears (Coix lacryma-jobi) - a critical review. Food Bioscience, 100537.

Dinh, D. M., Volpe, G. E., Duffalo, C., Bhalchandra, S., Tai, A. K., Kane, A. V., Wanke, A. C., \& Ward, H. D. (2014). Intestinal microbiota, microbial translocation, and systemic inflammation in chronic HIV Infection. Journal of Infectious Diseases, 211(1), 19-27.

Dizlek, H. (2015). Effects of amount of batter in baking cup on muffin quality International Journal of Food Engineering, 11(5), 629-640.

Fariña, M., Torres, M. D., \& Moreira, R. (2019). Starch hydrogels from discarded chestnuts produced under different temperature-time gelatinisation conditions. International Journal of Food Science and Technology, 54(4), 1179-1186.

Fenster, K., Freeburg, B., Hollard, C., Wong, C., Rønhave Laursen, R., \& Ouwehand, A. (2019). The production and delivery of probiotics: A review of a practical approach. Microorganisms, 7(3), 83.

Gaci, N., Borrel, G., Tottey, W., O'Toole, P. W., \& Brugère, J.-F. (2014). Archaea and the human gut: New beginning of an old story. World Journal of Gastroenterology, 20(43), 16062.

Ghoddusi, H. B., Grandison, M. A., Grandison, A. S., \& Tuohy, K. M. (2007). In vitro study on gas generation and prebiotic effects of some carbohydrates and their mixtures. Anaerobe, 13(5-6), 193-199.

Gibson, G. R., Hutkins, R., Sanders, M. E., Prescott, S. L., Reimer, R. A., Salminen, S. J., Scott, K., Stanton, C., Swanson, K. S., Cani, P. D., Verbeke, K., \& Reid, G. (2017). The International Scientific Association for Probiotics and Prebiotics (ISAPP) consensus statement on the definition and scope of prebiotics. Nature Reviews Gastroenterology \& Hepatology, 14, 491-502.

Gill, P. A., van Zelm, M. C., Muir, J. G., \& Gibson, P. R. (2018). Review article: Short chain fatty acids as potential therapeutic agents in human gastrointestinal and inflammatory disorders. Alimentary Pharmacology \& Therapeutics, 48, 15-34.

Goswami, D., Gupta, R. K., Mridula, D., Sharma, M., \& Tyagi, S. K. (2015). Barnyard millet based muffins: Physical, textural and sensory properties. LebensmittelWissenschaft und -Technologie- Food Science and Technology, 64(1), 374-380.

Heo, Y., Kim, M., Lee, J., \& Moon, B. (2019). Muffins enriched with dietary fiber from kimchi by-product: Baking properties, physical-chemical properties, and consumer acceptance. Food Sciences and Nutrition, 7(5), 1778-1785.
Hernot, D. C., Boileau, T. W., Bauer, L. L., Middelbos, I. S., Murphy, M. R., Swanson, K. S., \& Fahey, G. C. (2009). In vitro fermentation profiles, gas production rates, and microbiota modulation as affected by certain fructans, galactooligosaccharides and polydextrose. Journal of Agricultural and Food Chemistry, 57(4), 1354-1361.

Hoseney, R. C. (1994). Soft wheat products. Principles of cereal science and technology. Minnesota USA: American Association of Cereal Chemistry Inc. ISBN10, Article 0913250791.

Hunter, R., \& Harold, R. W. (1987). The measurement of appearance. New York USA: John Willey and Sons, ISBN 978-0-471-83006-1.

Kanjan, P., \& Hongpattarakere, T. (2017). Prebiotic efficacy and mechanism of inulin combined with inulin-degrading Lactobacillus paracasei I321 in competition with Salmonella. Carbohydrate Polymers, 169, 236-244.

Louis, P., Scott, K. P., Duncan, S. H., \& Flint, H. J. (2007). Understanding the effects of diet on bacterial metabolism in the large intestine. Journal of Applied Microbiology, 102(5), 1197-1208.

Lurie-Weinberger, M. N., \& Gophna, U. (2015). Archaea in and on the human body: Health implications and future directions. PLoS Pathogens, 11, Article e1004833.

Mohanty, D., Misra, S., Mohapatra, S., \& Sahu, P. S. (2018). Prebiotics and synbiotics: Recent concepts in nutrition. Food Bioscience, 26, 152-160.

Mordor Intelligence. (2020). Europe bakery products market - growth, trends, and forecasts (2020 - 2025). Internet: https://www.mordorintelligence.com/industry-re ports/europe-bakery-products-market . (Accessed September 2020).

Moreira, R., Chenlo, F., \& Torres, M. D. (2009). Simplified algorithm for the prediction of water sorption isotherms of fruits, vegetables and legumes based upon chemical composition. Journal of Food Engineering, 94(3-4), 334-343.

Morrison, D. J., \& Preston, T. (2016). Formation of short chain fatty acids by the gut microbiota and their impact on human metabolism. Gut Microbes, 7(3), 189-200.

Oswin, C. R. (1946). The kinetics of package life. Journal of the Society of Chemical Industry, 65(12), 419-421.

Ozato, N., Salto, S., Yamaguchi, T., Katashima, M., Tokuda, I., Sawada, K., Katsuragi, Y., Kakuta, M., Imoto, S., Ihara, K., \& Nakaji, S. (2019). Blautia genus associated with visceral fat accumulation in adults 20-76 years of age. Npj Biofilms Microbiomes, 5, 28.

Payne, A. N., Zihler, A., Chassard, C., \& Lacroix, C. (2012). Advances and perspectives in in vitro human gut fermentation modeling. Trends in Biotechnology, 30(1), 17-25.

Pérez-Ramos, A., Mohedano, M. L., López, P., Spano, G., Fiocco, D., Russo, P., \& Capozzi, V. (2017). In situ $\beta$-glucan fortification of cereal-based matrices by Pediococcus parvulus 2.6: Technological aspects and prebiotic potential. International Journal of Molecular Sciences, 18(7), 1588.

Pompei, A., Cordisco, L., Raimondi, S., Amaretti, A., Pagnoni, U. M., Matteuzzi, D., \& Rossi, M. (2008). In vitro comparison of the prebiotic effects of two inulin-type fructans. Anaerobe, 14(5), 280-286.

Power, S. E., O'Toole, P. W., Stanton, C., Ross, R. P., \& Fitzgerald, G. F. (2014). Intestinal microbiota, diet and health. British Journal of Nutrition, 111(3), 387-402.

Rinninella, E., Raoul, P., Cintoni, M., Franceschi, F., Miggiano, G., Gasbarrini, A., \& Mele, M. (2019). What is the healthy gut microbiota composition? A changing ecosystem across age, environment, diet, and diseases. Microorganisms, 7(1), 14

Rizzatti, G., Lopetuso, L. R., Gibiino, G., Binda, C., \& Gasarrini, A. (2017). Proteobacteria: A common factor in human diseases. BioMed Research International, 2017, 9351507.

Rodriguez-Colinas, B., Kolida, S., Baran, M., Ballesteros, A. O., Rastall, R. A., \& Plou, F. J. (2013). Analysis of fermentation selectivity of purified galacto-oligosaccharides by in vitro human faecal fermentation. Applied Microbiology and Biotechnology, 97(13), 5743-5752.

Rycroft, C. E., Jones, M. R., Gibson, G. R., \& Rastall, R. A. (2001). A comparative in vitro evaluation of the fermentation properties of prebiotic oligosaccharides. Journal of Applied Microbiology, 91(5), 878-887.

Sanz, T., Salvador, A., Baixauli, R., \& Fiszman, S. M. (2009). Evaluation of four types of resistant starch in muffins. II. Effects in texture, colour and consumer response. European Food Research and Technology, 229(2), 197-204.

Shevkani, K., \& Singh, N. (2014). Influence of kidney bean, field pea and amaranth protein isolates on the characteristics of starch-based gluten-free muffins. International Journal of Food Science and Technology, 49(10), 2237-2244.

Silvi, S., Verdenelli, M., Orpianesi, C., \& Cresci, A. (2003). EU project Crownalife: Functional foods, gut microflora and healthy ageing. Journal of Food Engineering, 56 (2-3), 195-200.

Sreenivas, K. M., \& Lele, S. S. (2013). Prebiotic activity of gourd family vegetable fibres using in vitro fermentation. Food Bioscience, 1, 26-30.

Szutowska, J. (2020). Functional properties of lactic acid bacteria in fermented fruit and vegetable juices: A systematic literature review. European Food Research and Technology, 246, 357-372.

Torres, D. P. M., Gonçalves, M. do P. F., Teixeira, J. A., \& Rodrigues, L. R. (2010). Galacto-oligosaccharides: Production, properties, applications, and significance as prebiotics. Comprehensive Reviews in Food Science and Food Safety, 9(5), 438-454.

Trinh, K. T., \& Glasgow, S. (2012). On the texture profile analysis test. Quality of life through chemical engineering, Chemeca 2012. September 2012, Wellington, New Zealand, pp. 749.

Zheng, J., Wittouck, S., Salvetti, E., Franz, C. M. A. P., Harris, H. M. B., Mattarelli, P., O'Toole, P. W., Pot, B., Vandamme, P., Walter, J., Watanabe, K., Wuyts, S., Felis, G. E., Gänzle, M. G., \& Lebeer, S. (2020). A taxonomic note on the genus Lactobacillus: Description of 23 novel genera, emended description of the genus Lactobacillus Beijerinck 1901, and union of Lactobacillaceae and Leuconostocaceae. International Journal of Systematic and Evolutionary Microbiology, 70, 2782-2858. 